EPJ Web of Conferences 71, 00072 (2014)

DOI: 10.1051/epjconf/20147100072

(C) Owned by the authors, published by EDP Sciences, 2014

\title{
Signs of hypothetical fauna of Venus
}

\author{
Leonid V. Ksanfomality ${ }^{1}$ \\ Space Research Institute, 84/32 Profsoyuznaya str., Moscow 117997 Russia
}

\begin{abstract}
On March 1 and 5, 1982, experiments in television photography instrumented by the landers VENERA-13 and -14, yielded 37 panoramas (or their fragments) of the Venus surface at the landing site. Over the past 31 years, no similar missions have been sent to Venus. Using a modern technique the VENERA panoramas were re-examined. A new analysis of Venusian surface panoramas' details has been made. A few relatively large objects of hypothetical fauna of Venus were found with size ranging from a decimeter to half meter and with unusual morphology. Treated once again VENERA-14 panoramic images revealed 'amisada' object about $15 \mathrm{~cm}$ in size possessing apparent terramorphic features. The amisada's body stands out with its lizard-like shape against the stone plates close by. The amisada can be included into the list of the most significant findings of the hypothetical Venusian fauna. The amisada's body show slow movements, which is another evidence of the Venusian fauna's very slow style of activity, which appears to be associated with its energy constraints or, and that is more likely, with the properties of its internal medium. The terramorphic features of the Venusian fauna, if they are confirmed, may point out at outstandingly important and yet undiscovered general laws of the animated nature on different planets.
\end{abstract}

\section{Introduction. Finds of the TV-VENERA experiments}

The results of a series of Soviet VENERA missions are the only existing observations of Venus' surface; they were conducted in 1975 and 1982 [1-4]. The experiments were of extreme technical complexity. Over the years, they have not been repeated by any Russian or other space agencies. Recently a new analysis of Venusian surface panoramas' details has been made. A few samples of the planet's hypothetical flora have been found.

The lander VENERA-13 worked longer than the others (127 min instead of 30 min guaranteed.) The coordinates of the lander landing site were $7.5^{\circ} \mathrm{S}, 303.5^{\circ} \mathrm{E}$, and its height above the level of radius $6051 \mathrm{~km}$ was $1.9 \mathrm{~km}[5]$. The temperature was $735 \mathrm{~K}\left(462^{\circ} \mathrm{C}\right)$ and the pressure was $8.87 \mathrm{MPa}$, which corresponds to the atmospheric density $59.5 \mathrm{~kg} / \mathrm{m} 3$, with the composition $\mathrm{CO} 2(96.5 \%)$ and N2 $(3.5 \%)$. The local time was 10:00 am, and the solar zenith angle was of $37^{\circ}$. Illumination by the diffused sunlight was 3-3.5 kLux.

\footnotetext{
${ }^{1}$ leksanf@gmail.com
}

This is an Open Access article distributed under the terms of the Creative Commons Attribution License 2.0, which permits unrestricted use, distribution, and reproduction in any medium, provided the original work is properly cited. 
The lander VENERA-14 sank at the equatorial zone at $13^{\circ} \mathrm{S}, 310^{\circ} \mathrm{E}$, and the landing site's height was $1.3 \mathrm{~km}$ above the radius of $6051 \mathrm{~km}$. The measured physical conditions were as follows: temperature $738 \mathrm{~K}$, pressure of $9.47 \mathrm{MPa}$ and atmospheric density approximately $65 \mathrm{~kg} / \mathrm{m} 3$. Gas analyzers repeated that the atmosphere is composed almost entirely of CO2 $(96,5 \%)$ and $\mathrm{N} 2(3.5 \%)$. Local time was also at approximately $10 \mathrm{am}$, with a solar zenith angle of $36^{\circ}$. The scene illumination reached 3.5 $\mathrm{kLx}[2,6]$. In both cases, the transmission of images began with a one-minute delay after landing to prevent any dust from obscuring the optical view.

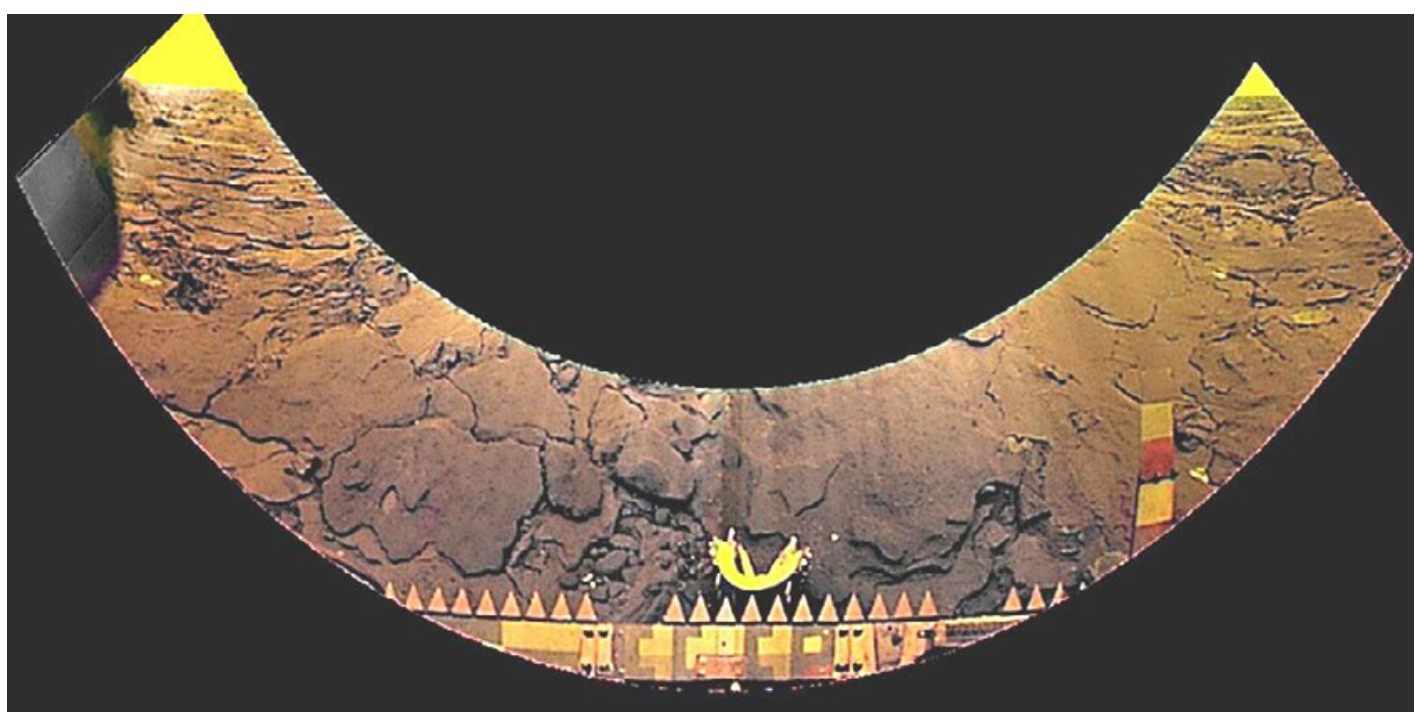

Figure 1. The Venus surface at the landing sites of VENERA-14. The VENERA panorama has been re-processed. Geometric distortions are corrected.

The thematic issue of "Kosmicheskiye Issledovaniya", V. XXI, No. 2-3, 1983, presented the main results of the VENERA-13 and -14 missions. The methodology of the television experiments on the surface of Venus and the date and list of the experimental data have been discussed in detail in $[1,2,4]$ and are not repeated here. Some remarks on the critical features of the treatment used are given below.

View of the planet's surface at the landing sites of both landers are shown in Figure 1. In the first case (VENERA-13), the view was crushed soil with stone slabs, and in the case of VENERA -14, it was plates of lithified deposits of dust or traces of ancient volcanic eruptions. Physical properties of the surface at the VENERA -14 landing site (March 1982) were presented in [7]. In many sites, the planet's surface is similar to solidified dust or lava. The daytime illumination attains $5--10 \mathrm{kLx}$ and higher; blue light is absorbed by the atmosphere, and the sky tint is yellowish. The Sun disk usually is not seen through permanently presenting clouds. The cloud layers are located high in the atmosphere and are composed of micron-size droplets of concentrated sulfuric acid. The duration of both the day and the night is 58.4 Earth days. A typical Venusian landscape is a waterless almost red-hot stony or loose surface, mountains and sometimes volcanoes.

The quest for hypothetical flora and fauna on Venus is based on analysis of the images of the landing site of the VENERA-13 and VENERA-14 landers. At the time of this writing, almost three years have elapsed since the submission of the first manuscript for publication that was devoted to hypothetical signs of life on the planet Venus [1]. An accumulation of new objects increased gradually (Figure 2). These objects hypothetically have characteristics of living creatures - flora or fauna. At the initial stage of the investigation, mainly panoramas of VENERA -9 and - VENERA -13 were used, in which relatively large objects were found, such as "scorpion", "mushroom", "bear" and "owl" [1, 2, 8, 9]. 


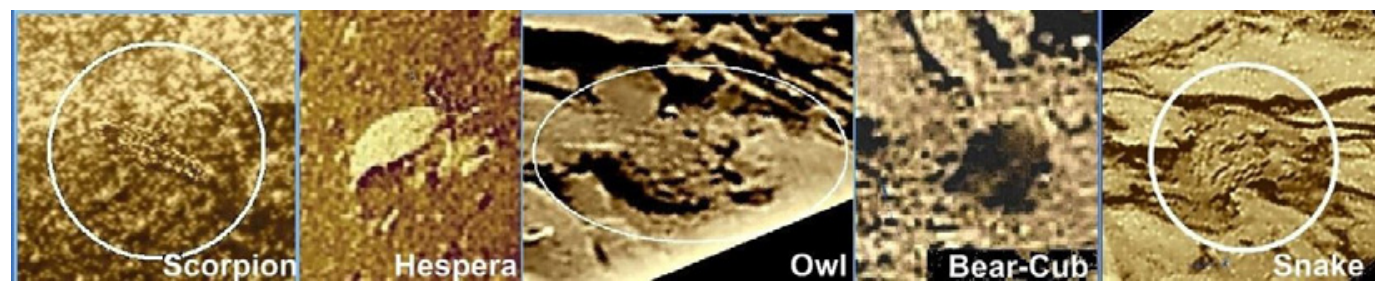

Figure 2. Gallery of entities described in $[1,2,7]$. All names are conditional.

When experience using image processing was accumulated, the VENERA-14 panorama allowed an approach to the finer details [7]. An important role was played by additional image processing, image geometric correction and the presence of up to eight duplicates of images that were obtained with good quality and low levels of noise. This arrangement enabled the selection and staking of their fragments. As a result, it managed to find and learn about a few new types of hypothetical inhabitants of the planet at the VENERA-14 landing site: "stems" and a "snake." Treated once again VENERA-14 panoramic images revealed 'amisada' object, about $15 \mathrm{~cm}$ in size possessing apparent terramorphic features.

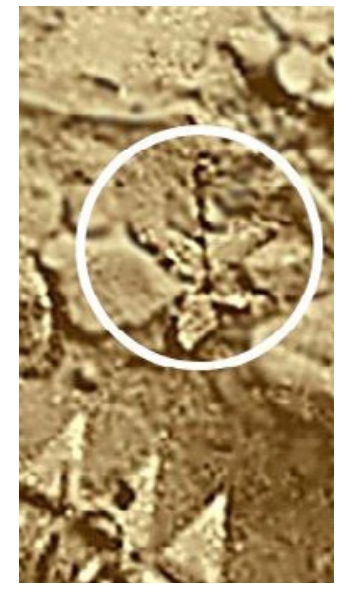

Figure 3. The first found "stem" is a thin vertically arranged knotty trunk that has a height of approximately 40 $\mathrm{cm}$ and a thickening ("burgeon") on the top and the "quatrefoil" at its base. The "stem " is located at a distance of approximately $0.4 \mathrm{~m}$ from the landing buffer of the VENERA-14 lander and is seen from above.

Despite this paper is devoted to Venusian fauna, few words should be said about its flora. Of importance is a question about the sources of energy for the hypothetical Venusian inhabitants. The interest in the proposed autotrophic flora of the planet as a source of the existence of its fauna was noted in [3, 8-11]. It is natural to assume that, like on the Earth, the Venusian fauna is heterotrophic, and the source of its existence is hypothetical autotrophic flora (produced in unknown type of photosynthesis). Although the direct rays of the sun, as a rule, do not reach the surface of the planet, there is enough light for photosynthesis of the Earth-like type there. In the case of the Earth, a diffuse illumination of 0.5-5 kLux is sufficient for photosynthesis even in the depths of the dense forests. The measured luminance on Venus is of the same order, at the range of 0.4 to 9 kLux. Of course, photosynthesis at high temperatures and in a non-oxidizing environment should be based on a completely different, unknown biophysical mechanism.

What was found are numerous vertically standing knotty black stems with heights of up to half a meter (Figure 3). Due to the availability of up to eight duplicates of the images obtained and their low level of masking noise, the VENERA panoramas permit identifying and exploring some new types of 
hypothetical life forms of Venus. Specifically, stems are the most numerous group of samples of hypothetical flora.

Still more surprising was another find. When processing the VENERA-13 images with a decreasing contrast, this allowed us to see a "flower" - an object of a regular shape (Figure 4), with a white spot (pestle?) in the center and the surrounding petals. The flower is composed of six to eight light petals. The "flower" size is approximately the same as a "quatrefoil" at the base of the stem.

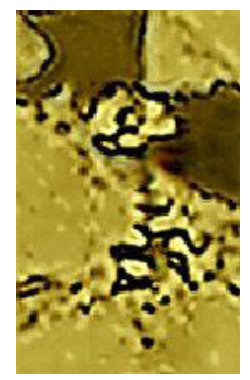

Figure 4. Flower - an object of the VENERA-13 panorama (with lowered contrast) that unveiled a detailed image of the "flower", its light central part and leaves at the base. The diameter of the flower and the "quatrefoil" at the base are $5-8 \mathrm{~cm}$.

The stems are an important complement to the objects of a hypothetical Venusian flora discussed in $[8,11]$. If there are Venusian flowers, one should reflect their role. The flowers of terrestrial plants are intended for their pollination and reproduction. Pollination is conducted either by insects or by the wind. Flowers attract insects. Do Venusian burgeons and flowers, at least indirectly, hint on the likely participants in the process of pollination?

\section{Objects "amisada" at the VENERA-14 landing site}

Pictures of the Venusian surface returned by VENERA landers contained images of unusual objects (found before the stems) that were not recognized immediately. This finding could indicate the existence of life on Venus, although at first glance, such a proposal sounds absurd. Analysis of the treated VENERA-14 panoramic images once again, revealed an object 'amisada' that stands out with an unusual shape against the stone plates that are close by. The 'amisada' can be included into the list of the most interesting findings of the hypothetical Venusian fauna. Among hypothetical fauna entities of Venus, which are presented in our previous papers [1-3, 8, 9], certain unusual findings that have similar structure were found in different areas of the planet. Their unusual shape was repeated on various panoramas that were taken by landers' cameras and have attracted attention. The present paper addresses another object, for which the nickname 'amisada' is proposed. This type of object is repeated on successive panoramas. At a cursory examination, when seen from above, its body has an elongated fish-like shape with a size of $12--15 \mathrm{~cm}$.

It should be underlined again that the physical conditions on Venus are incompatible with terrestrial life at all. The Venusian atmosphere is almost entirely composed of carbon dioxide, and cloud layers are composed of micron-size droplets of concentrated sulfuric acid. A hypothetical waterless Venusian life, if it does exist, must use biophysical mechanisms of metabolism and photosynthesis, which are distinguished from those of the Earth's life.

The studies published previously $[1-3,5]$ have reported on the positive results of searches for hypothetical forms of fauna and flora on Venus. The conclusions were based on the newly processed panoramic images of the Venusian surface, which had been obtained more than 30 years ago.

In total, there were 41 images or their fragments returned by the landers' cameras in 1975 and 1982 [2, 4]. Until now, 11 or 12 unusual objects were found, analyzed and discussed in relevant 
publications. The re-processing of the images made it possible to detect novel unusual objects.

The VENERA-14 hypothetical fauna objects 'amisada' were originally referred to as "hesperas" (similar to the case of the VENERA-13 landing site [12]). However, the unusually shaped objects apparently represent another hypothetical group of Venusian fauna than hesperas. Similar to [1-3] and for convenience of description, the specific nickname "amisadas" was proposed for this group of objects. (This name is an abbreviation for the ancient Babylonian title of clay tablets "Ammisaduqa», in which apparitions of Venus were recorded.)

The amisadas are described here for the first time. The natural question arises as to why no objects of this type were found previously. This fact can be explained by several reasons. The first reason is that nobody was looking for them, although the earliest suggestive publication about the subject is dated 1978 [13]. The second reason is the necessity to develop novel processing methods that did not exist previously, and the processing is very labor-consuming. It should be noted that the processing of primary images to the level that is required to search for hypothetical forms of Venusian fauna and flora as well as the search in itself are difficult and time-consuming tasks. These considerations are the facts that can explain why the systematic study that began four years ago, in 2009 , has resulted so far in 11 or 12 findings only of hypothetical objects. The amisadas are related to the most recent results. Until now, the analysis has not been completed yet; approximately half of the available images were studied.

The easiest result is the detection of terramorphic objects, such as a 'Bear-cub' [3]. Nevertheless, even in these cases, it took a long time (sometimes up to half a year) to recognize an object that was presented in an insufficiently clear image. Of course, the possibility of artifacts associated with the interference of noise cannot be excluded, especially when the images are noisy. To address these cases, a very thorough analysis was required.

\section{Amisada's raw images}

Images published soon after the completion of the VENERA missions were obtained on the basis of single or combined black-and-white or color-divided panoramas. As was reported, there are many primary raw images that have not been studied yet. (Detailed information on TV experiments on the VENERA-13 and VENERA-14 landers was published in $[2,4]$ and is not repeated here).

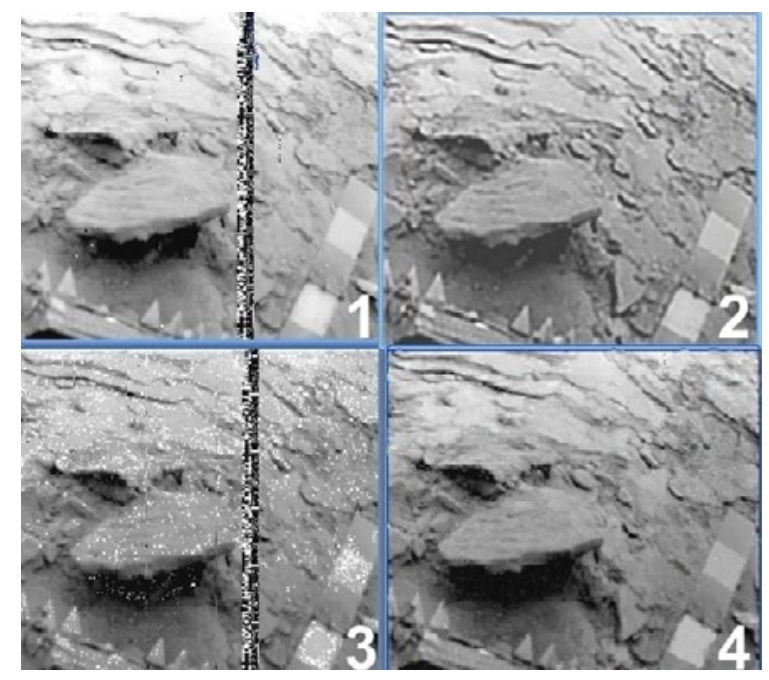

Figure 5. Illustration of primary-processing methods for selected fragments of Venusian surface panoramas: (1) and (3) are initial images at the VENERA-14 landing site (camera 1); (2) and (4) correspond to the insets that eliminated and partly eliminated noise. 


\section{EPJ Web of Conferences}

In contrast to cameras 1 of the VENERA-13 and VENERA-14 landers, their cameras 2 transmitted a full black-and-white image and then colored fragments of only the right and left parts of the panoramas; 14 fragments were taken by each camera. These images constitute four consecutive series of repeated groups of images, which are numbered 1, 6, 9 and 13. There is a contradiction for a history of the images. We have found that according to the arrangement and positions of the noise and defects, series 6 could be a retransmitted series 1 , and series 13 could be a retransmitted series 9 , although they have certain differences. Each series includes a number of panoramas. On the most successful images, the noise is low, which allows using efficient processing methods and combining different images by a skacking. As a result, the quality of the images was improved noticeably.

Figure 5 shows an example of the primary processing steps of the most successful panoramas for series $1 / 6$ and 9/13, in which the identified fragments were selected. Images 1 and 3, respectively, correspond to original fragments with very low (1) and middle (3) noise levels. The vertical band is telemetry data that has been inserted from other devices. In images 2 and 4, inserts are replaced by sections that were taken from other panoramas of the same series, and the noise interferences are partly removed. The fragments obtained admit further significant improvement in the sharpness and clarification of the fine details. To this end, four methods were employed: application of an unsharpmask and the functional-sharpness methods; use of the correlative-stacking operation; and an improvement in the sharpness by consistent application of the "blur-sharpening" operation of standard WORD and WINDOWS software. All of these approaches are known sufficiently well, and a significant number of relevant codes have been proposed for each of them.

\section{4 'Amisada' climbing a stone}

The most interesting 'amisada' was found in the central part of the VENERA-14 panorama. The amisada is located at the landing buffer and is marked by the white circle in Figure 6. This object is closest to the lens of camera 1 and is being observed from above, at an angle of approximately $80^{\circ}$ to the horizon. In contrast to other findings, the amisada is, apparently, seen in motion. Seemingly, it is climbing up or creeping on a 5-8-cm stone. The upper part of the amisada is located on the flat surface of the stone, and the lower part envelops a ledge.

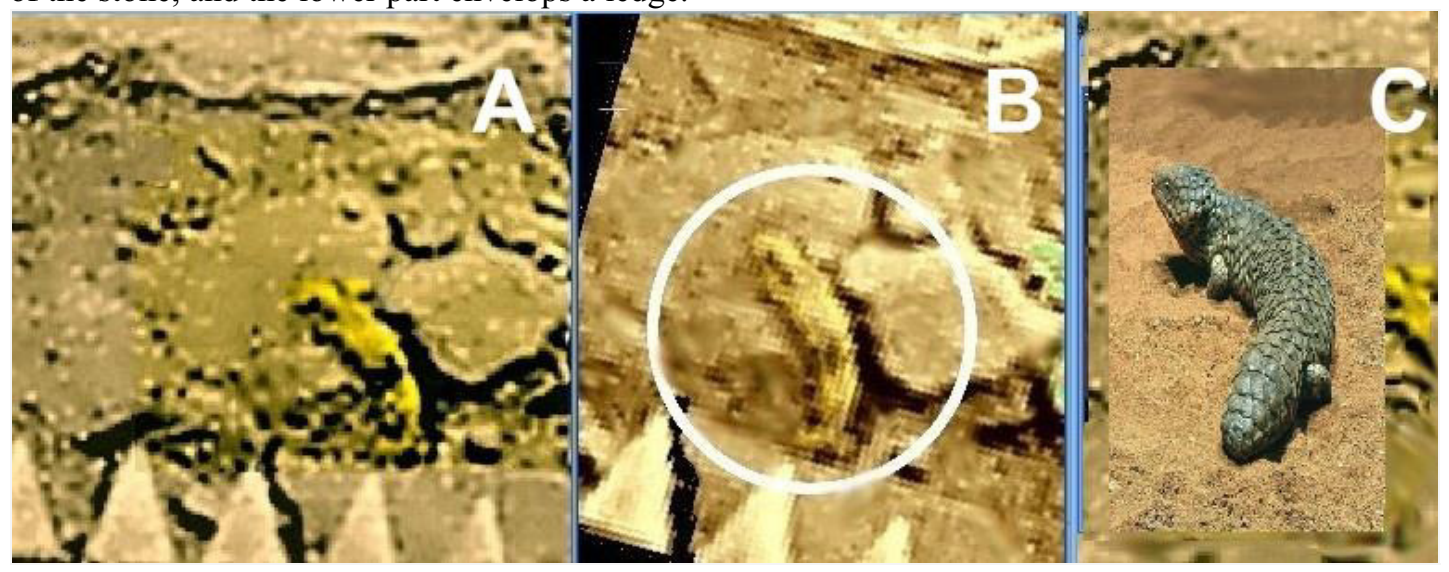

Figure 6. Single image of the spotty amisada (A); averaged view of 6 raw images (B); and the Earth' Australian lizard shingleback (C) similar to the amisada in size, extremities and tardiness. In its position, the amisada resembles a lizard climbing up on a stone. The slow displacement of the amisada's head (upper part) has been detected.

Of course, it cannot be excluded that amisada does not go up but instead comes down from the stone. However, from a comparison with other detailed images of the amisada shown below, its head is the upper part of Figure 6, and it is actually climbing up onto a stone. Figure 6 shows its single raw 
image (A) and averaged view (B) made by stacking of 6 raw images, together with an image of the Earth' Australian lizard shingleback (C) having approximately the same size and, may be, a shape. Two small protrusions at both sides of the amisada's head could be its forelimbs. The lower part of the amisada, its "tail", appears to be bifurcated. In general, the amisada's position resembles a lizard climbing up a rock, which again indicates the surprising terramorphism of Venusian fauna. There are distinctive spots on the amisada's body (Figure 6). Dark spanning bands are visible at its central (at the bend) and lower parts. The length of the amisada is approximately $12-15 \mathrm{~cm}$.

There are a few consecutive panoramas with the amisada. A more detailed treatment made it possible to trace the consecutive movements of the "head" portion and the likely amisada's "limbs" (Figure 7 and 8). In Figure 7, the amisada's front end and its left extremities are directed to the left (compared with Figure 6). In Fig. 7, the direction of its "head" part varies in frames 1-3 approximately in the direction of 10, 7 and 8 o'clock, and it repeats this type of motion in the less clear frames numbered 4-6. Additionally, the amisada's "forelimb" positions change. It is interesting to compare frames 1 and 3 of Figure 88. There are shadows on Venus surface that are cast by low-placed small objects only. In frame 3, the "head" part casts a clear shadow, but there is not such a shadow in frame 1. Therefore, we can assume that, at frame 1, the amisada's "head" is lifted up.

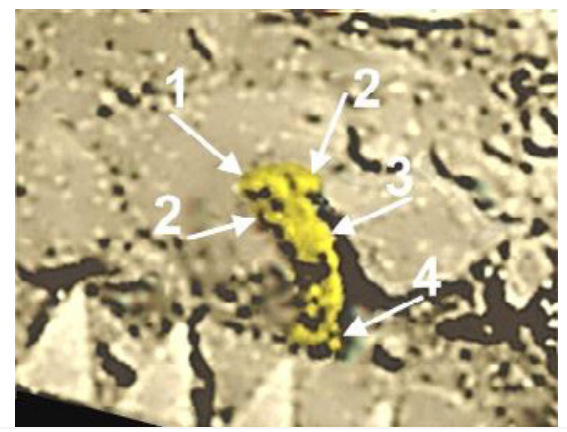

Figure 7. Amisada's third position (BW-2) at the panorama V-14-1-1. Arrow 1 shows the amisada's "head" part, the two arrows labeled 2 show the possible "front extremities", and the arrows labeled 3 and 4 show the body and tail of the amisada.

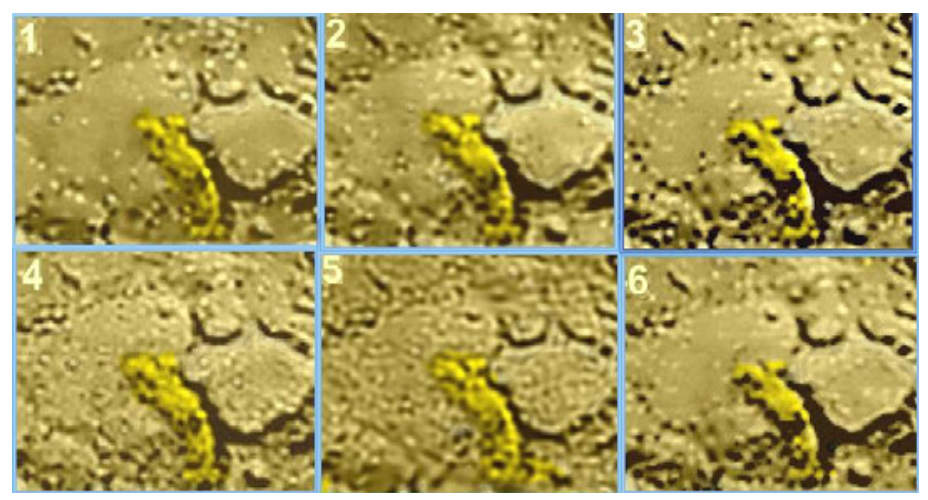

Figure 8. Consecutive positions of the "head" and "limbs" of the amisada at the panoramas V-14-1 1/6 and 9/13. Changes in the direction of its "head" part and in the positions of its "front extremities" are seen.

The animation of subsequent frames of the amisada (as shown in Figure 8) has made it possible to see a slight displacement of the light and dark parts of its head and of the protrusions. For 1.5 hours, the head gradually shifted first left for two pixels and then for two or three pixels, to opposite sides. At a distance of approximately $90 \mathrm{~cm}$ from the camera lens and for a resolution of 11', the displacement corresponds to $1.1-1.3 \mathrm{~cm}$. In this case, the speed of the motion is significantly lower than that 


\section{EPJ Web of Conferences}

determined in $[3,5]$. The speed is closer to $1 \mathrm{~mm} / \mathrm{min}$, rather than $1 \mathrm{~mm} / \mathrm{s}$. This value appears to be the result of a "housekeeping action" of the amisada (looking for food on the surface of the stone?) rather than its speed of motion.

Classification of amisadas as forms of hesperas, which had been proposed in $[2,5]$ for panoramas of VENERA-14, was likely incorrect. The body of a hesperos is flattened and is twice as long as that of an amisada. The amisada's body is thicker and flexible, as seen from Figure 6. Apparently, these were amisadas but not hesperas that had been shown in Figures 3 and 8 of [2,9], respectively.

\section{More distant amisades}

Results of the listed above operations (an unsharp-mask, sharpness of the fine details and of other less significant operations) are presented in Figure 9 for the series 1/6 (on the left) and for the series 9/13 (on the right). The improved clarity of the images made it possible to detect objects that were not recognized previously. The layered structure of the surface, with numerous cracks, is clearly seen. The color-control panel (on the right) provides the scale size, with each of its fields being a square of $10 \mathrm{x}$ $10 \mathrm{~cm}^{2}$. The distance between the teeth on the landing buffer is $5 \mathrm{~cm}$. Thus, the size of the large stone in the foreground is approximately $50 \mathrm{~cm}$.

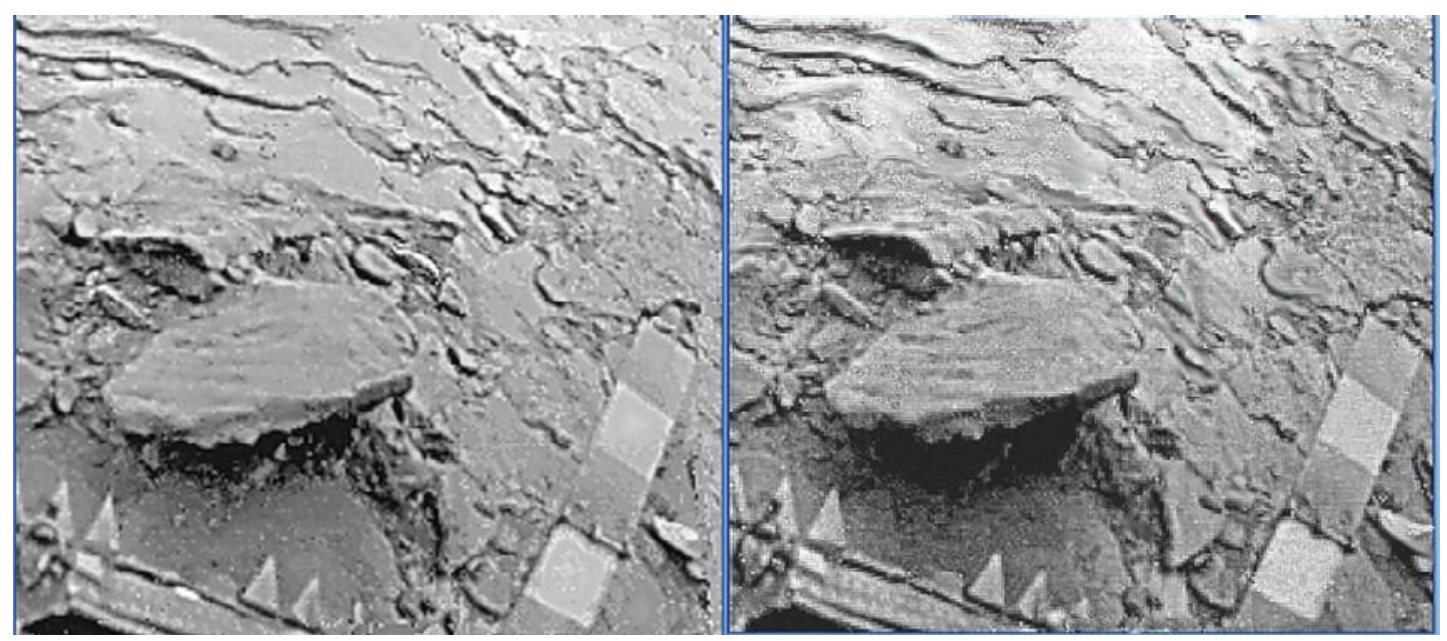

Figure 9. Advanced methods of processing Venusian surface panoramas improve their sharpness and make it possible to identify objects that were missed previously. On the left: processed image of the VENERA-14 panorama fragment (camera 1, series 1/6); on the right: the same fragment on panoramas of series 9/13.

The amisada- 1 object is located on the left of a large stone, in a small alcove at the left edge of the picture and is positioned favorably, being close to the camera lens at a distance of $1.2 \mathrm{~m}$. The distance between the amisada-1 and the spotty object shown in Fig. 3.4 is approximately $0.4 \mathrm{~m}$. The amisada-1 is seen at an angle of approximately $60^{\circ}$ to the horizon and represents an elongated bulky body, 12-15 $\mathrm{cm}$ long, which resembles a fish if observed from above. Its frontal part (on the left) terminates by a regular structure. As a result of the first attempt to clarify the amisada's structure, the image shown in Fig. 3.6 was obtained. The left side of the amisada is surrounded by a structure that resembles a tilted crown that is composed of individual non-resolved light elements. The regular structure of the "head" (left) part consists of isolated unresolved elements that form a round crown-like semi-circle that is salient from the amisada's body for $2--3 \mathrm{~cm}$. At the right, the amisada is terminated by a short narrow appendage that is similar to the hesperos' tail [4]. 


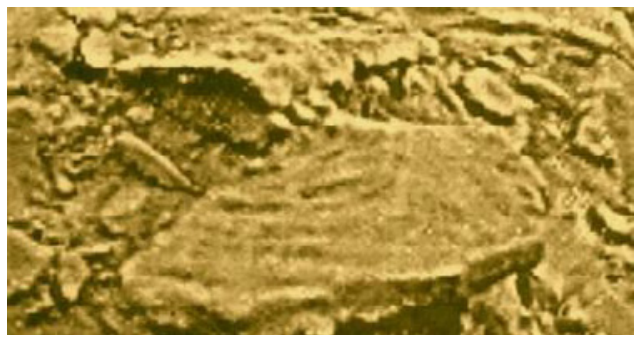

Figure 10. On the left: Amisada-1. The size of the object is approximately $15 \mathrm{~cm}$.

Of interest is the lower part of the amisada. According to the image, the amisada rests on the projecting parts of its body, and their number at this side can be three or five. The apparent inclined position of the body is explained by the geometry of the image, namely, by the $50^{\circ}$ tilt of the camera axis. Under the object, a deep shadow is seen, which indicates the volume character of this object.

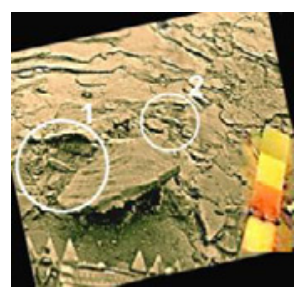

Figure 11. Positions of amisadas 1 and 2.

The detailed analysis of Figure 9 allows us to find the second amisada, which is located roughly twice as far as the first one. The positions of both amisadas are shown in Figure 11, in which they are marked by the white circles labeled 1 and 2. The amisada- 2 is arc-shaped and is seen also in Figure 9.

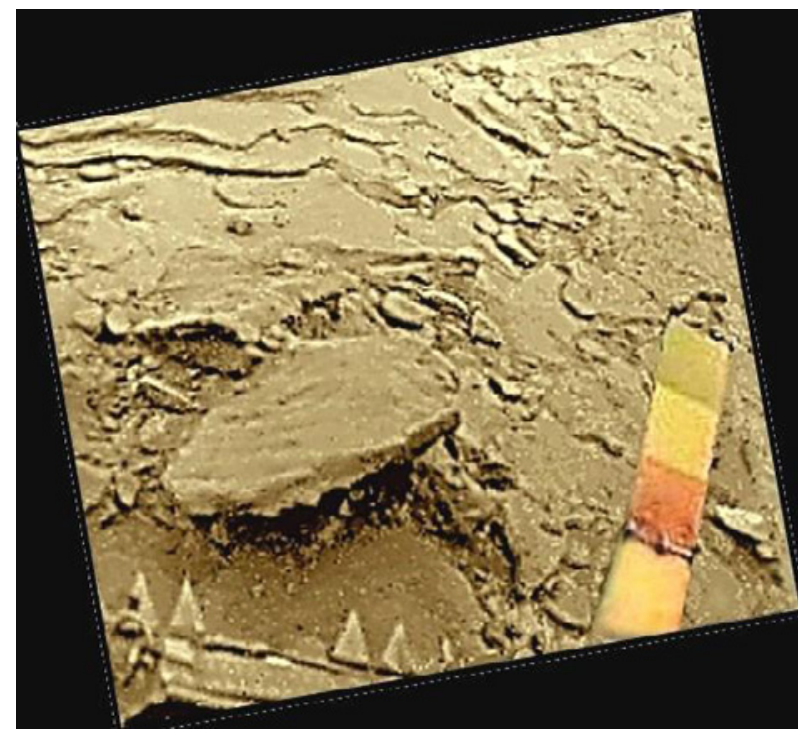

Figure 12. The high sharpness image stacked from two parts of Figure 9.

Combining parts of the images obtained independently, one arrives at the more detailed view of the fragment (Figure 12), where the geometry is corrected in part. Apparently, amisada-2 is slightly larger 


\section{EPJ Web of Conferences}

than the first amisada. During the observation time, the amisada-1 displaced slightly and the inclination of its "crown" changed, that was found by animation methods.

The "crown" that is clearly visible at the amisada-1 in Figure 10 is hardly seen (if any) in the object shown in Figure 6. Therefore, on the one hand, if the crown is considered to be a signature of the amisada- 1 and -2 head parts, then there is no firm proof that the features in Figures 6-8 belong to the same type of fauna. On the other hand, because each image line takes $0.78 \mathrm{~s}$, the blurring of the crown image could be caused by the wind above the stone surface (in contrast, both amisadas shown in Figures 9-12 were positioned in calm depressions).

The regular and similar shapes of the amisadas 1,2 and their mutual proximity underline their reality. In Figure 13, the views of amisadas 1 and 2 are compared at their positions in Figure 9. Due to the larger distance to the second amisada, its details are less distinguishable; only its tail and spots on the body are seen. The lower part of the amisada- 2 is not completely visible, but the similarity of the images for both of the amisadas appears to be evident. The crown actually belongs to these objects and extends their length up to $15 \mathrm{~cm}$. For amisada-2, its total length reaches approximately $15-17 \mathrm{~cm}$. Two more distant hypothetical amisadas are noticed at other parts of the VENERA-14 panoramas. Thus, at least three amisadas were found at the VENERA-14 landing site. However, shown in Figure 6 is the most interesting object.

The number of high-resolution BW-images is insufficient for a more detailed conclusion; for the color-divided panoramas, they included only the right part of the amisada-1. In any case, the estimate given in [3,5] for the maximum movement velocity that is characteristic of Venusian fauna, which was approximately $1 \mathrm{~mm} / \mathrm{s}$, is not exceeded here.

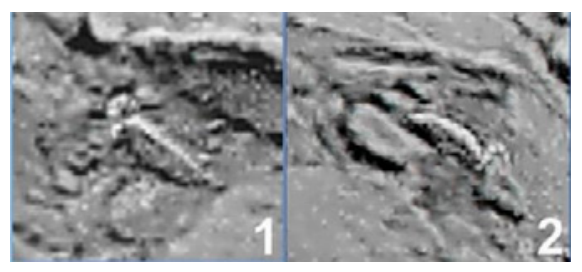

Figure 13. Comparison of shapes for amisadas-1 and -2. Probably the crown formed by individual elements does not have a random nature and relates to the amisadas themselves.

Amisadas were also found in the VENERA-13 panoramas. However, the objects were too far from the camera; as a result, the resolution of the image was insufficient to recognize the fine details.

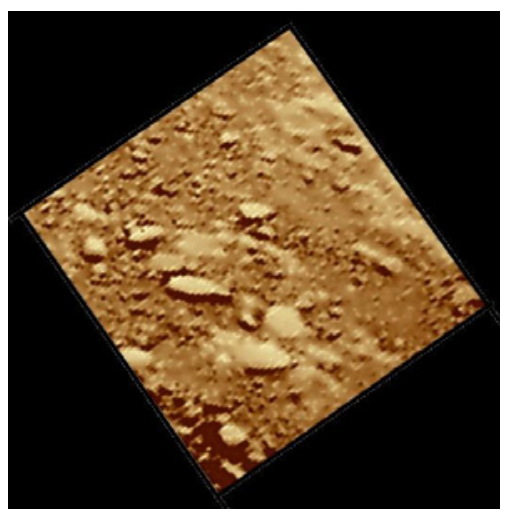

Figure 14. Result of the processing of fragments of the VENERA-13 panoramas upon employment of all of the available images.

The shape of the object was reconstructed by rigorous processing that used all of the available images, repeated in successive panoramas. The above-listed processing methods were applied. 
A small shift in the successive images within a single pixel results in an additional improvement in the resolution. Of course, the image cannot be better than that contained in the original images. Figure 14 illustrates the final results that were obtained from the step-by-step stacking of the images. Thus, one can assume that the elongated body shown in Figure 14 can actually be an amisada rather than a hespera. However, due to the large distance of the object from the camera lens, the resolution is insufficient for the detection of fine details, such as a crown, spots, and other characteristic features of the objects traced on the VENERA-14 panoramas (as shown in Figure 10).

\section{Conclusions}

The analysis of the VENERA-14 panoramas made it possible to reveal objects called 'stems' and 'amisada'. Both of them possess undeniable terramorphic features. The shapes of stems and of the amisada's body are clearly different from the background, the surrounding stone plates. The amisada can be referred to as one of the most interesting findings among the hypothetical Venus fauna. Another finding, a 'stem,' is of interest as well. If the tops of the stems really are burgeons and flowers, one should reflect their role. The flowers of terrestrial plants are intended for their pollination and reproduction. Therefore the stems and flowers of Venus, at least indirectly, hint on the likely participation of other organisms (insects?) in the process of their pollination.

The amisadas are presented to the reader for the first time. The object amisada resembles a lizard climbing up on a stone. The amisada- 1 and -2 have well-distinguished fish-shaped bodies and might be supplied by limbs for support (and, probably, for motion), similar to those of reptiles. The structure and purpose of the amisada body part that resembles a crown is not clear. The 'amisada' was found to move, although very slowly, which confirms once again to the data of [3,5] on the slow pattern of physical activity of the Venus fauna (as compared with the Earth fauna), which could be due to its energy constraints or due to nature of unknown active medium of their organisms, inasmuch as water does not exist on Venus in its liquid form. With respect to the terramorphic features of the Venus fauna $[3,7,9]$, in case they are confirmed, they would point at exceptionally important general laws of a living nature, yet undiscovered by science, occurring at absolutely different physical conditions, including the high-temperature, oxygen-free Venus environment or the settings of some types of exoplanets.

A limited fragment of the panorama, which is presented in the text, contains images of three amisadas. One might assume that they are widespread entities of the Venusian fauna. The variety of forms of hypothetical fauna and flora of Venus suggests a complex relationship among the detected objects. The VENERA TV-cameras were intended to produce a general notion about the planet's surface and did not anticipate problems finding possible inhabitants of Venus. The special mission, if it ever takes place, should be significantly more complex.

\section{REFERENCES}

1. L.V. Ksanfomality, Doklady Physics, 57, 367 (2012)

2. L.V. Ksanfomality, IJAA, 3, 57 (2013)

3. L.V. Ksanfomality, Doklady Physics, 58, 497 (2012)

4. A.S. Selivanov, Yu.M.Gektin, M.A.Gerasimov, B.I.Nosov, M.K.Naraeva, Kosmich. Issled., 21, $176(1983)$

5. L.V. Ksanfomality, Advances in Zoology and Botany 1, 25 (2013)

6. B.E. Moshkin, A.P. Economov, V.I. Moroz, Kosmich. Issled., 21, 236 (1983)

7. L.V. Ksanfomality, Doklady Physics, 58, 395 (2013)

8. L.V. Ksanfomality. The Hypothetical Flora of Venus. Doklady Physics, 58,196 (2013)

9 L.V. Ksanfomality, International Letters of Chemistry, Physics and Astronomy, 10, 76 (2013)

10. L.V. Ksanfomality, Doklady Physics, 58, 514 (2013) 
EPJ Web of Conferences

11. L.V. Ksanfomality, Doklady Physics, 58, 204 (2013)

12. L.V. Ksanfomality, Doklady Physics, 57, 455 (2012)

13. L.V. Ksanfomality, Planets Discovered Anew (Fizmatlit, Moscow, 1978). 